\title{
El contexto hospitalario infantil: el efecto de los payasos de hospital y otras intervenciones
}

\author{
Livia García Pérez y Diana Martín Luis \\ Universidad de La Laguna
}

\author{
María Luisa Mora Quintero \\ Hospital Universitario de Canarias
}

\section{RESUMEN}

En este trabajo se revisa la eficacia de las investigaciones basadas en las intervenciones de los payasos de hospital, terapia con mascotas y musicoterapia, para mejorar la situación de los niños en el hospital. Se seleccionaron 22 estudios encontrados en las bases de datos PubMed, EBSCOhost, Web of Science y CSIC, y 3 en referencias bibliográficas, de los cuales 13 utilizaron payasos de hospital, 6 incluyeron terapias con animales y 6 emplearon música. La mayoría de las intervenciones analizadas obtuvieron resultados positivos en la reducción de ansiedad, miedo, dolor, u otras variables. La revisión de los datos señala que la presencia de estas intervenciones en el hospital tiene un efecto positivo sobre la salud y que podrían ser usadas en distintos contextos terapéuticos. Estos resultados contribuyen a la comprensión de la importancia de aproximaciones no farmacológicas en la promoción del bienestar en el entorno hospitalario. Sin embargo, los problemas metodológicos encontrados apoyan la necesidad de seguir desarrollando intervenciones en esta línea.

Palabras clave: intervención; payasos de hospital; hospital; niños; ansiedad; malestar psicológico.

\begin{abstract}
This paper review the research on the effectiveness of research based on hospital clowns, pet therapy and music therapy interventions, to improve the situation of children in hospital. Twenty-two studies found in PubMed, EBSCOhost, CSIC and Web of Science and three of references were selected, of which 13 used hospital clowns, 6 included animal therapy and 6 used music. Most interventions showed positive results in reducing anxiety, fear, pain, or other variables. The data reviews indicate that these interventions in the hospital setting has a posible health-inducing effect, and could be used in differents therapeutic settings. These results contributes to the current understanding of the importance of non-pharmacological approaches in promoting well-being within hospital settings. However, methodological problems found support the need for further interventions.
\end{abstract}

Keywords: Intervention; hospital clown; hospital; child; anxiety; distress. 


\section{Introducción}

En el análisis de la emoción humana tradicionalmente se le ha dado más importancia a las emociones negativas que a las positivas. Aunque ambas no son excluyentes y se encuentran en la misma persona, deben estudiarse aisladamente (Ripoll \& Quintana, 2010). Entre los beneficios de las emociones positivas están, la mejora de la forma de pensar (Lyubomirsky, King, \& Diener, 2005) y el bienestar; que aparecen relacionados con la salud, con una mayor calidad de vida y con la supervivencia (Danner, Snowdon, \& Friesen, 2001); y que representan un apoyo frente a la adversidad (Fredrickson, 2003).

La investigación sobre el estrés ya desde los estudios clásicos de Selye (1974) ha descrito el impacto de las emociones negativas sobre la salud, tales como el miedo, la ira y la ansiedad. Ha sido más escasa la literatura que analiza las emociones positivas que pueden producir efectos beneficiosos en ella (Burgdorf \& Panksepp, 2006). Entre estos efectos se encuentran una mayor tolerancia al dolor, efecto analgésico, el estímulo del sistema inmune y la merma de las posibles consecuencias cardiovasculares nocivas de las emociones negativas (Bruehl, Carlson, \& McCubbin, 1993; Finan \& Garland, 2015; Fredrickson, 2000; Stoney, Cox, Valdimarsdottir, Jandorf, \& Neale, 1987).

La hospitalización infantil es un evento estresante, tanto para los niños como para sus familiares (Pelechano, 2008). Se puede determinar que entre el $10-35 \%$ de los niños que han pasado por la experiencia de estar hospitalizados presentan dificultades a la hora de volver a casa y un porcentaje que supera el $92 \%$ de ellos desarrolla crisis de conducta. Entre las respuestas más habituales que manifiestan los niños, se puede encontrar la ansiedad. Algunas de ellas se desarrollan con más magnitud cuando vuelven a casa. Entre las reacciones que manifiestan destacan el temor a la enfermedad, el sentimiento de ser ignorados o interpretar el entorno hospitalario como extraño y adverso. A esto habría que añadir el estrés generado ante los procesos quirúrgicos y la separación de los progenitores que viene aparejada con esta situación. De acuerdo con todo ello, promover el uso de estrategias favorecedoras de emociones positivas en la población infantil repercute en la mejora de la calidad de vida de los niños hospitalizados (Ortíz,2006).

Desde hace algunos años ha ido surgiendo un amplio número de estudios sobre el humor, y en concreto en niños, considerado como un moderador de situaciones estresantes o dolorosas y como un potenciador de la vitalidad y la recuperación de los pacientes. Algunos resultados apoyan los beneficios del humor y de la risa en áreas de rehabilitación cardíaca (Tan, Tan, Berk, Lukman, \& Lukman, 1997), percepción del dolor (Weisenberg, Raz, \&Hener, 1998), umbral de malestar (Cogan, Cogan, Waltz, \& McCue, 1987), afrontamiento del estrés (Martin \& Lefcourt, 1983) y mejora del sistema inmune (Bennett \& Lengacher, 2006; Lambert \& Lambert, 1995). Igualmente, las intervenciones con el humor han logrado una disminución en la ansiedad de los niños y un incremento en su colaboración en los distintos procedimientos médicos (Dowling, Hockenberry, \& Gregory, 2003), tales como en la implantación de un catéter permanente (Frankenfield, 1996) y en el 
trasplante de médula ósea (Gottlieb \& Portnoy, 1988).

Con todo ello ha ido creciendo el interés en la presencia de los payasos en las salas hospitalarias y en particular en las áreas de pediatría, como una estrategia de afrontamiento ante situaciones estresantes provocadas por la hospitalización; aumentando su seguridad, favoreciendo una mayor recuperación, potenciando la esperanza de los padres y aumentando la colaboración del personal sanitario (Linge, 2013). En general se subrayan beneficios tanto en términos somáticos como psíquicos.

A pesar de ello, los payasos no constituyen la única técnica en el ámbito hospitalario que persigue el bienestar de los más pequeños. La terapia asistida con animales aporta una nueva opción para tratar padecimientos físicos y emocionales (Cruz, 2009). La relación entre el paciente y los animales permite una mayor adaptación al entorno hospitalario. En niños, reduce la ansiedad y el estrés, mejora su atención, favorece la interacción verbal y su colaboración con el personal sanitario (Jofré, 2005).

Otra alternativa cuyo uso se viene defendiendo en el contexto hospitalario es la musicoterapia. Con esta técnica se favorece la expresión de emociones en el niño y le permite adaptarse a las nuevas situaciones que se generan en el hospital, así como repercute en la promoción de la capacidad para resolver sus propios conflictos (Serradas, 2006).

A pesar de la popularidad creciente de este tipo de intervenciones alternativas, se siguen destacando importantes limitaciones. En cuanto a las intervenciones que emplean payasos de hospital, las futuras investigaciones deben enfocarse a examinar y mejorar la fiabilidad y validez de las medidas que se emplean para analizar los resultados; así como ampliar el número de participantes, y contar, para su comparación, con un grupo control (Maanen, 2012).

Con respecto a las investigaciones sobre la terapia con animales, aunque abren un interesante abanico de posibilidades clínicas, aún se encuentran en una situación de escasa validez empírica. López-Cepero et al. (2014) señalan que la labor de proporcionar una base empírica sólida, en base a este tipo de intervenciones, continúa recibiendo una atención relativamente escasa.

En relación al estado actual de la investigación sobre la aplicación de la música en niños en el ámbito hospitalario, la revisión reciente de Wright, Adams y Vohra (2013) aborda su utilización para procedimientos dolorosos. Plantea que las investigaciones futuras deben potenciar el conocimiento sobre los efectos y la seguridad de la terapia, las poblaciones y procedimientos que pueden ser más beneficiosos, el mejor y más eficaz tipo de música, si es mejor aplicar la musicoterapia activa o pasiva, y el momento más adecuado para intervenir durante el procedimiento.

Son muchos los trabajos que demuestran los beneficios de este tipo de intervenciones para promover una mayor calidad de vida y bienestar infantil. Por todo ello, el presente trabajo se plantea como objetivo, una revisión teórica de distintos trabajos publicados sobre intervenciones no farmacológicas en la población infantil circunscrita al ámbito hospitalario. 


\section{Método}

\section{Búsqueda bibliográfica}

Se llevó a cabo el procedimiento de búsqueda de la bibliografía teniendo en cuenta diversas fuentes de información: a) revisión sistemática de la literatura de las bases de datos electrónicas: PubMed, EBSCOhost, Web of Science (WOS) y CSIC; utilizando como términos para la búsqueda en las tres primeras: clown " $Y$ " hospital, payasos " $Y$ " hospital, humour " $Y$ " hospital " $Y$ " children, clown " $Y$ " children, animal assistted therapy " $Y$ " hospital, pet therapy " $Y$ " children, music " $Y$ " hospital " $Y$ " children, music therapy " $Y$ " hospital; y para la última: payasos "Y" hospital, musicoterapia, niños " $Y$ " hospital, payasos " $Y$ " niños, terapia " $Y$ " animales, música " $Y$ " niños; b) consulta directa de libros procedentes de la biblioteca de la Universidad de La Laguna; d) revisión de referencias de los artículos recopilados.

Los criterios de selección utilizados para incluir los estudios en esta revisión comprenden: 1) estar basados en niños, 2) que estén hospitalizados o acudan al hospital para someterse a algún procedimiento, 3) que aborden intervenciones que empleen "payasos de hospital", "terapia con mascotas", o "musicoterapia”.

Se examinaron un total de 4752 publicaciones en PubMed ( $N=2164)$, EBSCOhost ( $N$ $=1635)$, Web of Science $(\mathrm{N}=800)$ y CSIC $(\mathrm{N}=$ 153). De los 110 artículos seleccionados para ser leídos, 88 fueron excluidos tras su revisión completa una vez que se verificó que incumplían alguno de los criterios de inclusión. A su vez, tres artículos fueron incluidos manualmente, siendo seleccionados tras la revisión de las referencias. Para una descripción más detallada de la estrategia de búsqueda ver la Figura 1.

Finalmente, 25 resultados fueron seleccionados para esta revisión; de los cuales, 13 abarcaron intervenciones con payasos, $6 \mathrm{em}$ plearon terapia con animales y otros 6 musicoterapia.

\section{Resultados}

\section{Descripción de los estudios}

En general, participaron un total de 1657 niños, con edades que oscilan entre 32 semanas (Standley et al., 2010) y 18 años (Sobo, Eng, \& Kassity-Krich, 2006), para aquellos trabajos que informaron del rango de edad de los participantes. Sin embargo, cabe destacar que uno de los estudios (Kingsnorth, Blain, \& McKeever, 2011) presenta un rango entre 4 y 21 años. Teniendo en cuenta que la muestra es pequeña ( $n=10$ ) y la media de edad es de 10.7 , no se ha tenido en cuenta la edad de 21 años como límite del rango.

En cuanto al origen de los participantes, en siete estudios pertenecen a EE.UU. (Braun, Stangler, Narveson, \& Pettingell, 2009; Golan, Tighe, Dobija, Perel, \& Keidan, 2008; Kaminski, Pellino, \& Wish, 2002; Kemper, Hamilton, McLean, \& Lovato, 2008; Sobo et al., 2006; Standley et al., 2010; Tsai, Friedmann, \& Thomas, 2010); en cinco estudios los integrantes son de Italia (Anastasi, Caprilli, Grotto, Scollo, \& Messeri, 2007; Bertini, Isola, Paolone, \& Curcio, 2011; Caprilli \& Messeri, 2006; Vagnoli, Caprilli, \& Messeri, 2010; Vagnoli, Caprilli, Robiglio, \& Messeri, 2005); de Canadá en cuatro trabajos (Barrera, Rykov, \& Doyle, 2002; Kingsnort et al., 2011; Wolyniez et al., 2013; Wu, Niedra, Pen- 
dergast, \& McCrindle, 2002); de España en tres trabajos (Gutiérrez et al., 2008; Meisel et al., 2009; Ortigosa, Gutierrez, Astilleros, Sánchez, \& Riquelme, 2011); de Perú en tres (Calmet, Regalado, \& Guevara, 2008; Rivara et al., 2010; Rivara et al., 2008); y en un estudio de Alemania (Pinquart, Skolaude, Zaplinski, \& Maier, 2011), Dinamarca (Hansen, Kibaek, Martinussen, Kragh, \& Hejl, 2011), e India (Balan, Bavdekar, \& Jadhav, 2009).

Respecto a las estrategias utilizadas, las técnicas más empleadas en las intervenciones con payasos de hospital son los trucos de magia (Bertini et al., 2011; Golan et al., 2008; Gutiérrez et al., 2008; Ortigosa et al., 2011; Pinquart et al., 2011; Vagnoli et al., 2010; Vagnoli et al., 2005; Wolyniez et al., 2013), la música
(Calmet et al., 2008; Golan et al., 2008; Kingsnorth et al., 2011; Pinquart et al., 2011; Vagnoli et al., 2010; Vagnoli et al., 2005), los juegos (Bertini et al.,2011; Calmet et al., 2008; Golan et al., 2008; Pinquart et al., 2011; Vagnoli et al., 2010; Vagnoli et al.,2005) y los títeres (Bertini et al.,2011; Golan et al., 2008; Gutiérrez et al., 2008; Ortigosa et al., 2011; Vagnoli et al., 2010; Wolyniez et al., 2013). También se emplean, aunque en menor medida, los muñecos (Calmet et al., 2008; Gutiérrez et al., 2008; Ortigosa et al., 2011; Vagnoli et al., 2005), las burbujas (Bertini et al.,2011; Golan et al., 2008; Vagnoli et al., 2005), los cuentos (Gutiérrez et al., 2008; Kingsnorth et al., 2011; Ortigosa et al., 2011), las bromas (Pinquart et al., 2011; Wolyniez et al., 2013), las obras de teatro y los globos (Cal-



Figura 1. Estrategia de búsqueda. Artículos revisados, excluidos y seleccionados. 
met et al., 2008), las sopas de letras (Vagnoli et al., 2010), los movimientos cómicos y los juegos con roles invertidos (Kingsnorth et al., 2011); así como, la imaginación y la realidad virtual (Wolyniez et al., 2013). Cabe destacar que hay tres estudios en los que no se definen las estrategias utilizadas (Hansen et al., 2011; Meisel et al., 2009; Rivara et al., 2010).

En cuanto a la terapia con animales, en todas las intervenciones se emplean perros, y en éstas, como estrategias, se recurre a que el paciente acaricie a la mascota (Kaminski et al., 2002; Sobo et al., 2006; Tsai et al., 2010), a que el animal vea con el niño la televisión, duerma al pie de la cama o a su lado, y haga diversos trucos como dar la pata (Sobo et al, 2006). También a que el paciente interactúe libremente con el animal (Caprilli \& Messeri, 2006; Wu et al., 2002); y a que la mascota permanezca en la cama o junto a la cama o silla donde esté el niño, dándole la oportunidad de cepillarlo (Tsai et al., 2010). Por otro lado, en uno de los estudios no se especifica la forma de actuación de la mascota (Braun et al., 2009).

Con respecto a los estudios que emplean música, en uno de ellos, los adolescentes y los niños en edad escolar se distraían con canciones de campamento, cantando, improvisando con los instrumentos, y escuchando música pregrabada, mientras que, los niños de preescolar y los más pequeños se dedicaban a reproducir canciones animadas, ritmos y a tocar instrumentos, como campanillas, tambores y maracas, entre otros (Barrera et al., 2002). En el resto de intervenciones, los músicos juegan, cantan y proponen juegos sonoros a los niños y a los padres (Anastasi et al, 2007); se emplean audífonos para intervenir con música clásica, reggaetón y salsa (Rivara et al., 2008); se emplea música desconocida para todos los participantes, utilizando el disco Heart Zones de Doc Childre (Kemper et al.,2008) así como música India (Balan et al., 2009), y canciones de cuna (Standley et al., 2010).

En relación con el momento en el que se realizan las intervenciones, en los procedimientos con payasos, en seis estudios se interviene en los momentos previos y de inducción de la anestesia antes de una intervención quirúrgica (Golan et al., 2008; Gutiérrez et al., 2008; Meisel et al., 2009; Ortigosa et al., 2011; Vagnoli et al., 2010; Vagnoli et al., 2005); en dos estudios durante la extracción de sangre (Rivara et al., 2010; Wolyniez et al., 2010); en uno durante los tratamientos con inyecciones de toxina botulínica (Hansen et al., 2011); y en cuatro de ellos no se interviene durante ningún procedimiento médico, sólo durante sesiones de risoterapia (Calmet et al., 2008), en sus habitaciones (Kingsnorth et al., 2011; Pinquart et al, 2011) o en las salas acondicionadas para la intervención de los payasos (Bertini et al., 2011).

Respecto a las intervenciones con perros, en una de ellas se aplica de forma específica, en el momento en el que los niños padecen dolor agudo postoperatorio (Sobo et al., 2006). Sin embargo, en el resto no se interviene durante procedimientos médicos concretos, sino que se actúa en las habitaciones (Tsai et al., 2010; Wu et al., 2002), en salas del hospital (Caprilli \& Messeri, 2006), o bien no se especifica el lugar de intervención (Braun et al., 2002; Kaminski et al., 2002).

En cuanto a los procedimientos con musicoterapia, en uno de ellos el niño podía tener una sesión cuando tuviese dificultades con algún 
procedimiento médico, como tomar medicación, punciones lumbares, análisis, etc. (Barrera et al., 2002). En dos de ellos se emplea la música, exclusivamente, durante extracciones de sangre (Anastasi et al., 2007; Balan et al., 2009); y en los tres restantes durante visitas rutinarias (Kemper et al., 2008), durante una campaña de despistaje de anemia (Rivara et al., 2008) y du- rante la alimentación por sonda nasogástrica en prematuros (Stanley et al., 2010).

En las Tablas 1, 2 y 3 se presentan estos estudios ordenados alfabéticamente e indicando la identificación del trabajo (autores y año de publicación), la muestra, las intervenciones, los resultados principales y los instrumentos utilizados para la medida de los cambios.

\section{Tabla 1}

Investigaciones que emplean payasos de hospital

\begin{tabular}{cccc}
\hline Muestra & & \\
Publicación & $\begin{array}{c}\text { (n, \% sexo predominante, } \\
\text { media de edad })\end{array}$ & Intervenciones
\end{tabular}

Bertini et al. (2011)
43 niños sin rango de edad documentado

$58.14 \%$ niños

Edad media:

$\mathrm{GE}=7.71(\mathrm{DT}=2.47)$

$\mathrm{GC}=7.54(\mathrm{DT}=2.06)$
$\mathrm{GE}=$ Intervención de los payasos en salas del hospital $(n=21)$

$\mathrm{GC}=$ Sin intervención de los payasos $(n=22)$
-En GE, disminuye la PAD, frecuencia respiratoria y temperatura; revelando una reducción general del estrés.

- Descenso general del nivel de sufrimiento.
- The Wong/Baker pain rating scale FACES - Self-Evaluation Numeric Scale (NRS-11). - The Children's Hospital of Eastern Ontario Pain Scale - Medidas fisiológicas
Calmet et al. 30 niños entre 6-12 años (2008)

$$
\begin{aligned}
& \% \text { sexo }=\text { n.d. } \\
& \text { Edad media }=\text { n.d. }
\end{aligned}
$$

GE = intervención de los payasos durante sesiones de risoterapia

$\mathrm{GC}=$ n.d.
-En el GE el llanto, la - Guía de Observación enuresis, las pesadillas y la (incluye características inapetencia disminuyeron psicológicas y sociales). y la alegría aumentó drásticamente.
- modified-Yale

Preoperative Anxiety Scale - State-Trait Anxiety preoperatoria en Inventory for Children. comparación con los otros - Grabaciones en vídeo. grupos.

-Este alivio, en el GE, en la entrada a quirófano e inducción de la anestesia, es menos evidente.
Gutiérrez et al. 60 niños entre 6-10 años (2008)
GE = intervención de payasos antes de una operación $(n=30)$

$(n=30)$
W-No se alcanza significación estadística en ninguna de las - State-Trait Anxiety Inventory for Children. - Escala Analógica Visual medidas, pero se observan - Posthospital Behavior tendencias a tener menor Questionnaire ansiedad y miedo. 
Tabla 1

Investigaciones que emplean payasos de hospital (continuación)

Hansen et al. 60 niños entre 0-15 años (2011

$56.67 \%$ niños

Edad media: 4 (DT=n.d)
$\mathrm{GE}=$ Intervención de payasos durante el tratamiento de toxina botulínica

$(\mathrm{n}=$ n.d. $)$

$\mathrm{GC}=$ Sin intervención $(\mathrm{n}=$ n.d.)
-El efecto del payaso está - Medidas temporales relacionado con el género relacionadas con el llanto del paciente. Solo las niñas infantil ante inyección. se benefician.

-Efecto negativo en niños menores de 8 años.
Kingsnorth et 10 niños entre 4-21 años

$\%$ sexo $=$ n.d.

Edad media $=10.7$

(DT $=5.00)$ al. (2011)
GE = Intervención de payasos en las habitaciones $(n=10)$

$\mathrm{GC}=$ Sin intervención $(\mathrm{n}=10)$

*n $=10$ porque todos los niños pasan por ambas condiciones.
-8 niños mostraron cambios fisiológicos en respuesta a los payasos.

-Aumento significativo en la risa, sonrisa y descenso del dolor.

-Tendencia general a un estado de ánimo positivo en GE.

Meisel et al. $\quad 61$ niños entre 3-12 años (2009)

\section{$86.89 \%$ chicos}

Edad media $=6(\mathrm{DT}=2.55) \mathrm{GC}=$ sin intervención

Ortigosa et al. 79 niños entre 6-12 años (2011)

$54.43 \%$ niños

Edad media $=7.81$

$(\mathrm{DT}=1.73)$ $(n=33)$

GE = intervención de los payasos antes de una operación $(n=28)$

GE = Intervención de payasos antes de operación $(n=41)$

$\mathrm{GC}=$ Sin intervención $(\mathrm{n}=38)$
-No se encontró efecto significativo de los payasos en las conductas desadaptativas.
- Medidas de respuestas fisiológicas

- Feeling Face Cards

- Observación de

respuestas

- Entrevista semiestructurada (niños)
- Posthospital Behavior Questionnaire

- Facial Affective Scale - Entrevista estructurada sobre el miedo a los payasos (padres).

- State-Trait Anxiety

-En el GE no hay diferencias significativas entre el momento pretest y postest, mientras que en GC si aumenta significativamente la ansiedad.

-Niños del GE tienen una intensidad de respuesta similar en sala de espera y antesala de quirófano.

-Niños del GC están más alterados a los 7 días después de la operación.
Pinquart et al. 100 niños entre 6-14 años (2011)

$$
\begin{aligned}
& 56 \% \text { niños } \\
& \text { Edad media: } \\
& \text { GE }=10.0(\text { DT }=2.3) \\
& G C=9.5(D T=2.4)
\end{aligned}
$$

$\mathrm{GE}=$ Intervención de los payasos en las habitaciones $(\mathrm{n}=50)$

$\mathrm{GC}=$ Sin intervención $(n=50)$
-En el GE, el bienestar psicológico mejora desde el punto de vista de niños y padres

-También, en el GE, el bienestar físico mejora desde el punto de vista de los padres, pero no se puede interpretar como efecto de los payasos.
- KINDL-R, versión para niños y padres.

- Physical Well-being Scale - Psychological Well-being Scale 


\section{Tabla 1}

\section{Investigaciones que emplean payasos de hospital (continuación)}

-No se encuentran

efectos positivos en el bienestar psicológico en las siguientes 4 horas de la intervención.

Rivara et al. (2010)
68 niños entre 2-16 años con dos rangos de edad, 2-6 y 7-16.

$54.41 \%$ niños

Edad media:

- Niños de 2-6 años

$(\mathrm{GE}=3.44 ; \mathrm{GC}=3.79)$

- Niños de 7-16 años
$\mathrm{GE}=$ Intervención de dos payasos durante venopunción $(\mathrm{N}=30)$

$\mathrm{GC}=$ Sin intervención $(\mathrm{n}=38)$
-Disminución significativa - The Children's Hospital del dolor en el GE en los dos of Eastern Ontario Pain rangos de edad. Scale

- Escala Analógica Visual

-Diferencias significativas en - Self Assessment Scale la frecuencia cardiaca, solo - Medidas fisiológicas en niños de 7 a 16 años.

-Mayor saturación de oxígeno en el momento de la venopunción en GE.

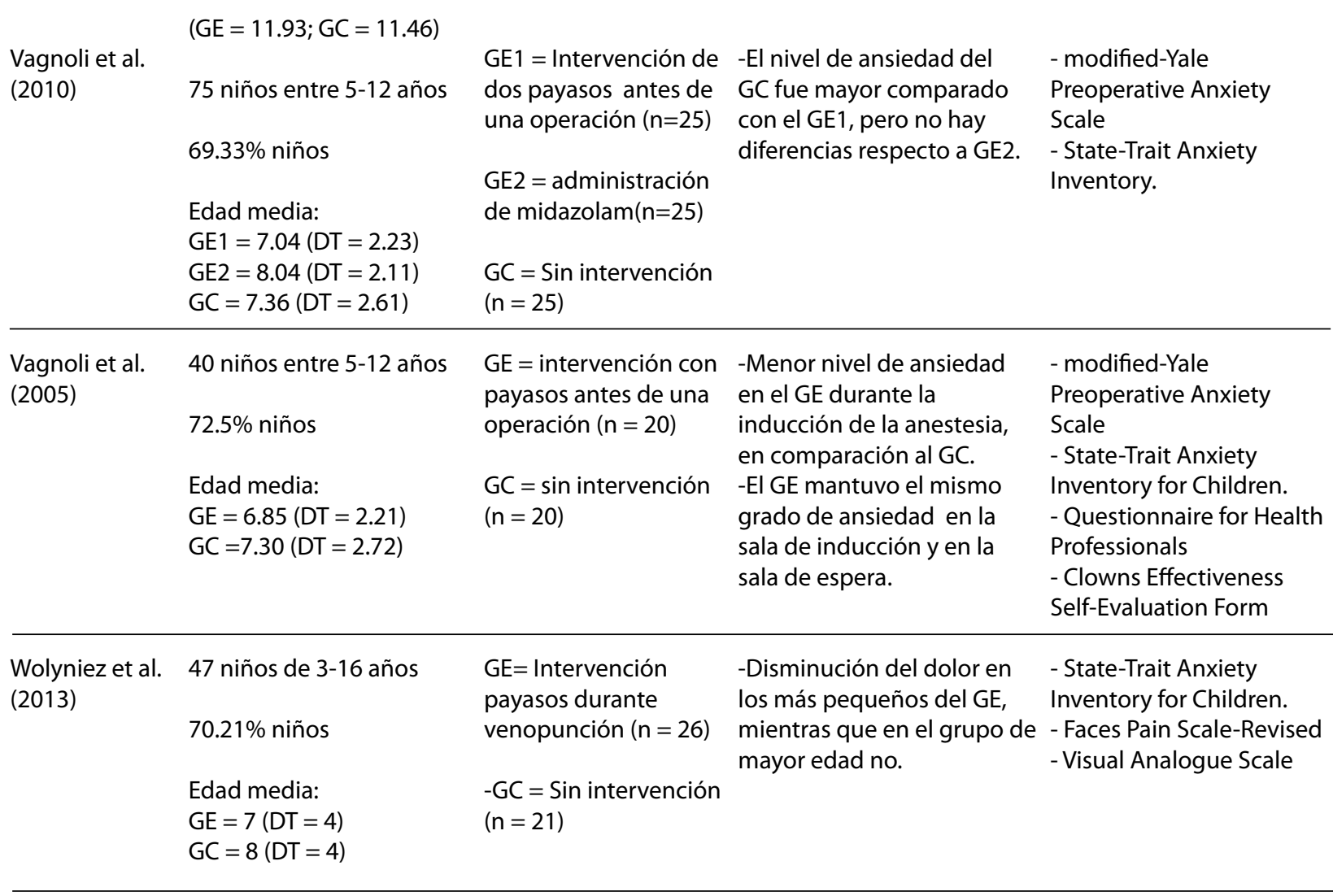

Nota. GE = grupo experimental; GC = grupo control; n.d. = no hay datos; $\mathrm{DT}$ = desviación típica. 
Tabla 2

Investigaciones que emplean terapia con mascotas

\begin{tabular}{|c|c|c|c|c|}
\hline Publicación & $\begin{array}{c}\text { Muestra } \\
\text { (n, \% sexo predominante, } \\
\text { media de edad) }\end{array}$ & Intervenciones & Resultados & Medidas \\
\hline
\end{tabular}

\begin{tabular}{|c|c|c|c|c|}
\hline $\begin{array}{l}\text { Braun et al. } \\
(2009)\end{array}$ & $\begin{array}{l}57 \text { niños entre } 3-17 \text { años } \\
50.9 \% \text { niños } \\
\text { Edad media = } 12.1 \text { (DT } \\
=4.4 .)\end{array}$ & $\begin{array}{l}\text { GE = Intervención de } \\
\text { perros }(n=18) \\
\text { GC = sin intervención } \\
(n=39)\end{array}$ & $\begin{array}{l}\text {-La presión arterial sistólica } \\
\text { era mayor en el GC al iniciar } \\
\text { el estudio } \\
\text {-Menor dolor en GE } \\
\text {-La presión arterial } \\
\text { y el pulso no varió } \\
\text { significativamente en el GE } \\
\text {-Las tasas respiratorias } \\
\text { fueron mayores en GE }\end{array}$ & $\begin{array}{l}\text { - FACES pain scale } \\
\text { - Medidas fisiológicas }\end{array}$ \\
\hline $\begin{array}{l}\text { Caprilli \& } \\
\text { Messeri (2006) }\end{array}$ & $\begin{array}{l}138 \text { niños en los } \\
\text { intervalos: } \\
1-3 ; 7-11 ;>11 \text { años } \\
\% \text { sexo = n.d. } \\
\text { Edad media }=3.5 \\
\text { (DT = n.d.) }\end{array}$ & $\begin{array}{l}\text { GE }=\text { Intervención de } \\
\text { perros en salas del } \\
\text { hospital ( } n=n . d .) \\
\text { GC = sin intervención } \\
(n=\text { n.d.) }\end{array}$ & $\begin{array}{l}\text {-Más beneficio en niños } \\
\text { hospitalizados por un largo } \\
\text { periodo. } \\
\text {-Los niños describen la } \\
\text { experiencia de terapia } \\
\text { asistida con animales como } \\
\text { positiva }\end{array}$ & $\begin{array}{l}\text { - The Self-Assessment } \\
\text { Manikin } \\
\text { - Escalas conductuales } \\
\text { - Análisis de los dibujos } \\
\text { de los niños }\end{array}$ \\
\hline $\begin{array}{l}\text { Kaminski et al. } \\
(2002)\end{array}$ & $\begin{array}{l}70 \text { niños mayores de } 5 \\
\text { años } \\
56 \% \text { niños } \\
\text { Edad media }=9.86 \\
(D T=2.80)\end{array}$ & $\begin{array}{l}\text { GE }=\text { Intervención de } \\
\text { perros }(n=30) \\
\text { GC = Sin intervención } \\
(n=40)\end{array}$ & $\begin{array}{l}\text {-Más afecto positivo en el } \\
\text { GE } \\
\text {-Mayor frecuencia cardiaca } \\
\text { en GE tanto antes como } \\
\text { después de la terapia. }\end{array}$ & $\begin{array}{l}\text { - Reynolds Child } \\
\text { Depression Scale (7 } \\
\text { ítmes) } \\
\text { - Padres y cuidadores } \\
\text { evaluaron estado de } \\
\text { ánimo de los niños. } \\
\text {-Grabaciones en vídeo de } \\
\text { interacción mascotas } \\
\text { - Indicadores fisiológicos } \\
\text { - Medida cortisol en } \\
\text { saliva. }\end{array}$ \\
\hline $\begin{array}{l}\text { Sobo et al. } \\
(2006)\end{array}$ & $\begin{array}{l}25 \text { niños entre } 5-18 \text { años } \\
64 \% \text { niñas } \\
\text { Edad media = n.d. }\end{array}$ & $\begin{array}{l}\text { GE = Intervención } \\
\text { de perros en periodo } \\
\text { de dolor agudo } \\
\text { postoperatorio } \\
(\mathrm{n}=25) \\
\mathrm{GC}=\text { no existe }\end{array}$ & $\begin{array}{l}\text {-Temas identificados en } \\
\text { las entrevistas: el perro } \\
\text { proporciona distracción, } \\
\text { placer, compañía, es } \\
\text { entretenido, hace recordar } \\
\text { al perro que se tiene en } \\
\text { casa, el niño disfruta con } \\
\text { el contacto del perro, es } \\
\text { tranquilizante y alivia el } \\
\text { dolor. } \\
\text {-La tasa de respuesta es alta }\end{array}$ & $\begin{array}{l}\text { - Escala de dolor y } \\
\text { malestar para niños } \\
\text { - Método de análisis del } \\
\text { habla desarrollado en } \\
\text { Psiquiatría (Gottschalk y } \\
\text { Gleser, 1969). } \\
\text { - Entrevista post- } \\
\text { intervención. }\end{array}$ \\
\hline
\end{tabular}


Tabla 2

Investigaciones que emplean terapia con mascotas (continuación)

Tsai et al. $\quad 15$ niños entre 7-17 años (2010)

\begin{tabular}{|c|c|}
\hline $53.3 \%$ niñas & habitaciones $(n=15)$ \\
\hline $\begin{array}{l}\text { Edad media: } \mathrm{n} . \mathrm{d} \\
\text { (DT = n.d.) }\end{array}$ & $\begin{array}{l}-\mathrm{GC}=\sin \text { intervención } \\
(\mathrm{n}=15)\end{array}$ \\
\hline & $\begin{array}{l}{ }^{*} \mathrm{n}=15 \text { porque todos } \\
\text { pasan por las dos } \\
\text { condiciones }\end{array}$ \\
\hline
\end{tabular}

\author{
-Las disminuciones en la - State-Trait Anxiety \\ presión arterial sistólica Inventory for Children. \\ siguieron después de que - Child Medical Fear Scale \\ la intervención hubiese - Monitor de presión \\ acabado. \\ sanguínea oscilométrico \\ Dinamap. \\ -La frecuencia cardiaca y la - Respuestas fisiológicas \\ presión arterial diastólica de niños. \\ aumentaron desde el \\ inicio hasta "durante" la \\ intervención en ambos \\ grupos.
}

\begin{tabular}{|c|c|c|c|c|}
\hline $\begin{array}{l}\text { Wu et al. } \\
(2002)\end{array}$ & $\begin{array}{l}30 \text { niños entre } 3 \\
\text { meses- } 16 \text { años } \\
67 \% \text { niños } \\
\text { Edad media = } 7 \\
\text { (DT = n.d.) }\end{array}$ & $\begin{array}{l}\text { GE = Intervención } \\
\text { de perros en las } \\
\text { habitaciones } \\
(\mathrm{n}=30) \\
\mathrm{GC}=\text { no existe }\end{array}$ & $\begin{array}{l}\text {-Cambios significativos en } \\
\text { los niveles de saturación de } \\
\text { oxígeno, tasas cardiacas y } \\
\text { respiratorias. } \\
\text {-31\% de los niños } \\
\text { informaron que la } \\
\text { presencia de los perros } \\
\text { habían ayudado a } \\
\text { normalizar su experiencia } \\
\text { de hospitalización; y el } \\
61 \% \text { informan que la } \\
\text { intervención supuso una } \\
\text { distracción. }\end{array}$ & $\begin{array}{l}\text { - Medida del nivel de } \\
\text { satisfacción con el } \\
\text { programa. } \\
\text { - Se evaluó el nivel } \\
\text { de interacción con la } \\
\text { mascota: actividad, } \\
\text { compenetración } \\
\text { sentimiento, impacto } \\
\text { medio ambiental, } \\
\text { beneficios. } \\
\text { - Medidas fisiológicas. }\end{array}$ \\
\hline
\end{tabular}

Nota. $\mathrm{GE}$ = grupo experimental; GC = grupo control; $\mathrm{n} . \mathrm{d}$. = no hay datos; $\mathrm{DT}$ = desviación típica. 
Tabla 3

Investigaciones que emplean musicoterapia

\begin{tabular}{|c|c|c|c|c|}
\hline Publicación & $\begin{array}{c}\text { Muestra } \\
\text { (n, \% sexo predominante, } \\
\text { media de edad) }\end{array}$ & Intervenciones & Resultados & Medidas \\
\hline $\begin{array}{l}\text { Anastasi et al. } \\
(2007)\end{array}$ & $\begin{array}{l}108 \text { niños entre } 4-13 \\
\text { años } \\
51.85 \% \text { niñas } \\
\text { Edad media }=4.8 \\
(D T=3.3)\end{array}$ & $\begin{array}{l}\text { GE = intervención } \\
\text { de música durante } \\
\text { venopunción }(n=54) \\
\text { GC = sin música } \\
(n=54)\end{array}$ & $\begin{array}{l}\text {-Efecto significativo de la } \\
\text { música en el dolor del GE. } \\
\text {-La angustia de los niños } \\
\text { del GE se ve afectada } \\
\text { significativamente } \\
\text { por la música antes, } \\
\text { durante y después de la } \\
\text { venopunción. }\end{array}$ & $\begin{array}{l}\text { - The Amended Form of } \\
\text { the Observation Scale } \\
\text { of Behavioral Distress } \\
\text { (OSBD-A). } \\
\text { - The Wong/Baker pain } \\
\text { rating scale FACES. }\end{array}$ \\
\hline $\begin{array}{l}\text { Balan et al. } \\
(2009)\end{array}$ & $\begin{array}{l}150 \text { niños entre 5-12 } \\
\text { años } \\
58.70 \% \text { niños } \\
\text { Edad media = } 7.96 \text { (DT } \\
=2.18 \text { ) }\end{array}$ & $\begin{array}{l}\text { GE1: intervención } \\
\text { con música durante la } \\
\text { venopunción }(n=50) \\
\text { GE2: utilización de } \\
\text { anestésicos locales } \\
(n=50) \\
\text { GC = sin música; } \\
\text { placebo }(n=50)\end{array}$ & $\begin{array}{l}\text {-La utilización de } \\
\text { anestésicos locales y } \\
\text { musicoterapia reducen el } \\
\text { dolor significativamente, } \\
\text { siendo más efectivos los } \\
\text { anestésicos locales. } \\
\text {-El placebo es menos eficaz } \\
\text { que la musicoterapia }\end{array}$ & - Visual Analog Scale \\
\hline
\end{tabular}

\begin{tabular}{|c|c|c|c|c|}
\hline $\begin{array}{l}\text { Barrera et al. } \\
(2002)\end{array}$ & $\begin{array}{l}65 \text { niños entre } 6 \\
\text { meses- } 17 \text { años } \\
50.77 \% \text { niñas } \\
\text { Edad media }=7 \\
(D T=4.80)\end{array}$ & $\begin{array}{l}\text { GE }=\text { Intervención } \\
\text { utilizando } \\
\text { musicoterapia ante } \\
\text { dificultades con } \\
\text { procedimientos } \\
\text { médicos } \\
\text { GC = no existe }\end{array}$ & $\begin{array}{l}\text {-En pre-escolares y } \\
\text { adolescentes, el juego } \\
\text { realizado después de la } \\
\text { intervención tendió a } \\
\text { mejorar, pero en niños de } \\
\text { edad escolar no. } \\
\text {-La mayoría de las } \\
\text { respuestas subjetivas de } \\
\text { los padres y niños fueron } \\
\text { positivas. }\end{array}$ & $\begin{array}{l}\text { - The Play-Performance } \\
\text { Scale } \\
\text { - FACES pain scale } \\
\text { - Cuestionario de } \\
\text { satisfacción de los autores } \\
\text { para padres (PSQ), niños } \\
\text { (CSQ) y personal sanitario } \\
\text { (SSQ). }\end{array}$ \\
\hline
\end{tabular}

Kemper et al. 47 niños, sin rango de (2008)

edad documentado
Pacientes que completan $\quad(n=47)$ la Escala Analógica Visual para ambas visitas $=60 \%$ niños; Edad media $=10.4$ (DT = 5.1)

Pacientes que completan Variabilidad del Ritmo Cardíaco para ambas visitas = 53\% niños; Edad media $=9.5$ (DT $=4.9$
$\mathrm{GE}=$ visita 2; atención

habitual + descanso en una habitación tranquila + música

-Mayor relación y menor nivel de frecuencia alta en el GE.

-No hay mayor efecto de la música sobre el bienestar subjetivo. (tenían altos GC = visita 1; atención habitual + descanso en una habitación tranquila el inicio) $(n=47)$

${ }^{*} \mathrm{n}=47$ porque todos pasan por ambas condiciones
- Visual Analog Scale - Medida de la tasa cardíaca 
Tabla 3

Investigaciones que emplean musicoterapia (continuación)

\begin{tabular}{|c|c|c|c|c|}
\hline $\begin{array}{l}\text { Rivara et al. } \\
(2008)\end{array}$ & $\begin{array}{l}150 \text { niños entre } 2-11 \\
\text { años. } \\
54 \% \text { niños } \\
\text { Edad media: } \\
\text { GE: G1=5.22; G2 = 5.07; } \\
\text { G3 = 5.00 } \\
G C=5.26\end{array}$ & $\begin{array}{l}\text { GE= Intervención } \\
\text { durante campaña de } \\
\text { despistaje de anemia } \\
\text { con música de } 3 \text { autores: } \\
\text { Mozart (GrupoA; } n=40) \text {, } \\
\text { Daddy Yankee (GrupoB; } \\
\mathrm{n}=39 \text { ) y Marc Anthony } \\
\text { (GrupoC; } n=37) \\
-\mathrm{GC}=\text { sin música } \\
(\mathrm{n}=34)\end{array}$ & $\begin{array}{l}\text {-No se encontraron } \\
\text { diferencias significativas } \\
\text { en cuanto a la frecuencia } \\
\text { cardíaca y la saturación de } \\
\text { oxígeno. } \\
\text {-La salsa tiene mayor efecto } \\
\text { analgésico en los niños } \\
\text { entre } 7 \text { y } 11 \text { años; y parece } \\
\text { que la clásica en niños } \\
\text { entre } 2 \text { y } 6 \text { años. } \\
\text { - The Children's Hospital of } \\
\text { Eastern Ontario Pain Scale } \\
\text { - Self-Assessment Scale } \\
\text {-The Kruskal-Wallis Test } \\
\text { - Medidas fisiológicas }\end{array}$ & $\begin{array}{l}\text { - The Children's Hospital } \\
\text { of Eastern Ontario Pain } \\
\text { Scale } \\
\text { - Self-Assessment Scale } \\
\text {-The Kruskal-Wallis Test } \\
\text { - Medidas fisiológicas }\end{array}$ \\
\hline $\begin{array}{l}\text { Standley et al. } \\
\text { (2010) }\end{array}$ & $\begin{array}{l}68 \text { niños entre } 32-36 \\
\text { semanas } \\
51.47 \% \text { niñas } \\
\text { Edad media: } \text { n.d. (DT = } \\
\text { n.d.) }\end{array}$ & $\begin{array}{l}\text { GE = intervención } \\
\text { con música durante } \\
\text { alimentación por sonda } \\
\text { nasogástrica } \\
\text { ( } n=45) \\
\quad \text {-GE1: recibe un } \\
\text { ensayo ( } n=24) \\
\quad-G 2 \text { recibe } 3 \text { ensayos } \\
(n=21) \\
\text { GC }=\text { sin música } \\
(n=23)\end{array}$ & $\begin{array}{l}\text {-Los tres ensayos } \\
\text { empleando el chupete } \\
\text { musical (GE) son más } \\
\text { efectivos que un solo } \\
\text { ensayo. } \\
\text { - El chupete musical es más } \\
\text { eficaz cuando se introduce } \\
\text { a las } 34 \text { semanas de } \\
\text { gestación }\end{array}$ & $\begin{array}{l}\text { - Encuesta } \\
\text { cumplimentada por } \\
\text { enfermeras ciegas al } \\
\text { propósito del estudio. } \\
\text { - Medidas de peso y otras } \\
\text { variables físicas. }\end{array}$ \\
\hline
\end{tabular}

Nota: $\mathrm{GE}=$ grupo experimental; GC = grupo control; n.d. = no hay datos; DT = desviación típica. 
Análisis de los resultados de los estudios

\section{Payasos de hospital}

Las intervenciones con payasos, de acuerdo con los resultados alcanzados, se podrían dividir en tres grupos. Por una parte, dos investigaciones no encuentran resultados significativos, a pesar de que se observan tendencias positivas hacia una menor ansiedad y miedo (Gutiérrez et al., 2008), así como, una fuerte tendencia a la disminución de conductas desadaptativas una semana después de la intervención quirúrgica (Meisel et al., 2009).

Por otra parte, cuatro intervenciones hallan resultados favorables, aunque únicamente para algunas de las condiciones evaluadas. En uno de los estudios el efecto del payaso está relacionado con el género, ya que únicamente las niñas se beneficiaron de la intervención realizada por la mujer que ejercía de payaso, frente a los niños que tuvieron efectos negativos (Hansen et al., 2011). En otro, se produjo un efecto positivo en el bienestar psicológico a corto plazo, pero éste desaparece a las 4 horas y no se produce ningún efecto en el bienestar físico percibido (Pinquart et al., 2011). Otra investigación concluye que los payasos son útiles para reducir el dolor y la ansiedad; pero a pesar de que existe una tendencia a la mejora del dolor, especialmente en los niños más pequeños, la mayoría de las diferencias no alcanzaron significación estadística (Wolyniez et al., 2013). Y por último, en la cuarta intervención se reduce, significativamente, la ansiedad en el preoperatorio, pero no es así durante la inducción de la anestesia (Golan et al., 2008).

Finalmente, siete investigaciones demuestran una clara efectividad de los payasos, al- canzando menores niveles de ansiedad durante la inducción de la anestesia (Vagnoli et al., 2010; Vagnoli et al., 2005); diminución del Ilanto, la enuresis, las pesadillas y la inapetencia frente a un aumento drástico de la alegría, de la comunicación con los iguales, con el personal sanitario, y con los padres, así como un incremento de la colaboración en procedimientos terapéuticos y juegos (Calmet el al., 2008); disminución de la frecuencia cardíaca junto con un aumento de la saturación de oxígeno (Rivara et al., 2010); disminución del dolor (Bertini et al., 2011; Kingsnorth et al., 2011; Rivara et al., 2010); reducción del estrés (Bertini et al., 2011); mantenimiento de la ansiedad, desde momentos previos hasta después de la cirugía, prolongándose su efecto más allá del periodo prequirúrgico (Ortigosa et al., 2011); así como un aumento de la risa (Kingsnorth et al., 2011).

En estas intervenciones se observan una serie de limitaciones. No se miden las diferencias entre el tiempo de inducción de la anestesia con y sin payasos (Vagnoli et al., 2010; Vagnoli et al., 2005); no se evalúan las diferencias de sexo, y sesgos de los evaluadores (Meisel et al., 2009); el tamaño muestral es pequeño (Bertini et al., 2011; Vagnoli et al., 2010; Wolyniez et al., 2013); falta de información sobre el comportamiento semanas después del alta (Vagnoli et al., 2010); dificultad para realizar procedimientos estandarizados con payasos (Bertini et al., 2011; Pinquart et al., 2011); falta de medidas e influencia de experiencias previas (Hansen et al., 2011), no se asignan aleatoriamente los sujetos a las condiciones (Meisel et al., 2009); surgen dificultades con los instrumentos de evaluación (Ortigosa et al., 2011); se evalúan 
parámetros subjetivos solo 4 horas después de la visita del payaso y se utiliza una muestra heterogénea (Pinquart et al., 2011); así como que pacientes y padres no fueron ciegos a la intervención, no se controló el uso de anestésicos locales y se limitaron los datos a intentos de canulación (Wolyniez et al., 2013). A esto podríamos añadir que las muestras de estos estudios son fundamentalmente masculinas.

\section{Terapia con mascotas}

Respecto a los estudios con animales, todos documentan resultados favorables, destacando el aumento del estado de ánimo y del afecto positivo (Kaminski, et al., 2002); efectos tanto estimulantes como relajantes con cambios apropiados en la frecuencia respiratoria y cardíaca (Wu et al., 2002); la percepción de que es una experiencia positiva, así como que contribuye a la ausencia de infecciones (CapriIli \& Messeri, 2006); aumento de la distracción (Sobo et al., 2006; Wu et al., 2002); se relaciona con incrementos de la sensación placentera, con sentirse acompañado, con entretenimiento y tranquilidad (Sobo et al., 2006); disminución del dolor (Braun et al., 2009; Sobo et al., 2006); además de menor presión arterial sistólica, manteniéndose en bajos niveles tiempo después de la finalización de la intervención (Tsai et al., 2010). A pesar de ello, en otros estudios se informa de que estas terapias producen un aumento de la frecuencia respiratoria (Braun et al., 2009), cardíaca (Kaminski et al., 2002; Tsai et al., 2010) y de la presión arterial diastólica (Tsai et al., 2010), que, sin llegar a definir estos resultados como potencialmente negativos, se atribuyen al entusiasmo genera- do por la actividad y la interacción de los pacientes con las mascotas.

Entre las limitaciones de estos estudios se reconocen la no inclusión de diversos tipos de pacientes y no controlar otras variables que puedan estar afectando los resultados (Kaminski et al., 2002); aspectos como el carácter voluntario del programa, la heterogeneidad de las edades y la realización de entrevistas conjuntas de padres e hijos (Wu et al., 2002); problemas con los instrumentos de medida (Kaminski et al., 2002; Wu et al., 2002); necesidad de más información (Caprilli \& Messeri, 2006); escaso tamaño muestral y medidas subjetivas (Sobo et al., 2006); falta de comparación con otros perros terapéuticos y no determinar la duración del alivio del dolor (Braun et al., 2009); así como bajo estrés y ansiedad previos a la intervención (Tsai et al., 2010).

\section{Musicoterapia}

Respecto a las intervenciones con música, se encontraron cuatro estudios que señalan resultados favorables en todas las condiciones evaluadas, documentándose comentarios subjetivos más positivos de niños y padres después de la intervención, así como mayor compromiso en actividades posteriores en preescolares y adolescentes (Barrera et al., 2002); disminución de la angustia y el dolor (Anastasi et al., 2007); menor dolor mediante el empleo del género "salsa" en niños entre 7-11 años y menor dolor, aunque no significativo, mediante el género "clásico" en niños entre 2-6 años (Rivara et al., 2008); así como menor dolor, mediante la música clásica hindú (Balan et al., 2009). Por otra parte, dos estudios encuen- 
tran resultados positivos en solo algunas de las condiciones, con aumentos en la relajación, aunque sin efecto sobre el bienestar subjetivo. Esto último se ha atribuido por una parte, a que los pacientes tenían un elevado bienestar antes de la intervención y, por otra, a que los informes de los padres pueden no detectar pequeños cambios en el bienestar (Kemper et al., 2008) y acortamiento de la duración de la alimentación nasogástrica solo en prematuros de 34 semanas (Stanley et al., 2010).

Finalmente, se revisaron las distintas medidas utilizadas en la detección de los cambios físicos y emocionales de los niños en las distintas intervenciones analizadas. Se presentan aquellos que han sido utilizados con una mayor frecuencia en los estudios analizados y que han sido capaces de detectar los cambios habidos con las intervenciones. Distintos estudios utilizaron para medir el miedo, la Escala Analógica Visual (Balan, et al, 2009; Gutiérrez et al., 2008; Ortigosa et al., 2011; Rivara et al., 2010; Wolyniez et al., 2013). Se aplicaron distintos tipos de medidas fisiológicas (Bertini et al., 2011; Braun et al., 2009; Hansen et al., 2011; Kaminski et al., 2002; Kingsnorth et al., 2011; Rivara et al., 2010; Wu et al., 2002). Para evaluar el dolor, FACES (Anastasi et al., 2007; Barrera et al., 2002; Bertini et al., 2011; Braun et al., 2009; Wolyniez et al., 2013); y The Children's Hospital of Eastern Ontario Pain Scale (Bertini et al., 2011; Rivara et al., 2010; Rivara et al., 2008). La ansiedad fue evaluada por los cuestionarios State-Trait Anxiety Inventory for Children (Gutiérrez et al., 2008; Ortigosa et al., 2011; Tsai et al., 2010; Vagnoli et al., 2005; Wolyniez et al., 2013); el Posthospital Behavior Questionnaire (Gutiérrez et al., 2008; Meissel et al., 2009; Ortigosa et al., 2011); y el Modified-Yale Preoperative Anxiety Scale (Golan et al., 2008; Vagnoli et al., 2010; Vagnoli et al., 2005).

En conclusión, como limitaciones de estos estudios se alude a la falta de control de otras variables, a la heterogeneidad muestral, a las medidas subjetivas, y a la falta de participación e información del personal sanitario (Barrera et al., 2002); a las medidas pretest tomadas con la intervención en curso y al sesgo del evaluador (Anastasi et al., 2007); al hecho de abordar el efecto de un tipo de música con una muestra poco homogénea (Kemper et al., 2008); y a no evaluar puntuaciones de dolor previas a la venopunción (Balan et al., 2009).

\section{Discusión}

En los últimos años ha habido un interés creciente en el estudio y desarrollo de las emociones, tanto positivas como negativas, y en sus repercusiones sobre la salud. En el área más concreta de la hospitalización, esta situación genera, en un gran número de casos, ansiedad y malestar psicológico. Esto se ve agravado en el caso de la hospitalización infantil, que puede llevar a sufrir alteraciones psicológicas. Esto ha promovido, en los últimos años, el aumento de técnicas de intervención dirigidas a dar respuesta a esta situación. En base a ello, el objetivo de esta revisión fue analizar la eficacia de las intervenciones de payasos de hospital, terapia con mascotas y musicoterapia en niños en situación hospitalaria. Sin olvidar que hay una carencia de estudios empíricos con resultados concluyentes y que, en ciertos casos, los 
trabajos publicados muestran deficiencias metodológicas de consideración.

El análisis de los resultados señala que, a pesar de que en algunos estudios solo se encuentran resultados favorables para algunas de las condiciones evaluadas, la proporción de hallazgos positivos es superior a los resultados imprecisos para algunas condiciones. Tan solo dos estudios de los evaluados no haIlaron significación empleando la intervención con payasos; sin embargo, consideran que es una técnica potencial que debe seguir siendo analizada y estudiada con rigor en posteriores investigaciones (Gutiérrez et al., 2008; Meisel et al., 2009).

La revisión de los distintos estudios plantea la necesidad de centrar el esfuerzo en garantizar una mayor fiabilidad en las medidas de evaluación, lo que permitiría una mejor detección de los cambios habidos en el estado emocional de los niños, con posibles repercusiones en la mejora de estas intervenciones (Maanen, 2012; Lopez-Cepero et al., 2014; Wright et al., 2013). Intervenciones que han demostrado ser potencialmente favorables para suavizar el impacto negativo que el entorno hospitalario tiene en la población infantil.

Por ello, el esfuerzo debe centrarse en mejorar las limitaciones que se han presentado en los estudios analizados. Prestando atención a la mejora de aspectos como el escaso tamaño muestral (Bertini et al., 2011; Sobo et al., 2006; Vagnoli et al., 2010; Wolyniez et al., 2013); la falta de medidas (Balan et al., 2009; Hansen et al., 2011; Vagnoli et al., 2005); la excesiva utilización de parámetros subjetivos (Barrera el al., 2002; Pinquart et al., 2011; Sobo et al.,
2006); la utilización de muestras heterogéneas (Barrera et al., 2002; Pinquart et al., 2011; Wu et al., 2002); las distintas dificultades con los instrumentos (Kaminski et al., 2002; Wu et al., 2002), y con la realización de procedimientos estandarizados (Bertini et al., 2011; Pinquart et al., 2011); la influencia de los sesgos (Anastasi et al., 2007; Meisel et al., 2009; Wolinyez et al., 2013); la limitación a un solo tipo de estrategia (Kemper et al., 2008; Kaminski et al., 2002); y la falta de control de algunas variables (Barrera et al., 2002; Kaminski et al., 2002). A esto uniríamos la necesidad de potenciar investigaciones que analicen la intervención de payasos con muestras más equitativas en cuanto al sexo.

Por lo tanto, a pesar de que es evidente que la contribución de estas iniciativas, da cabida a mayores progresos en el bienestar de la población infantil, es necesario que las futuras intervenciones solventen los problemas surgidos. Teniendo en cuenta que los resultados obtenidos hasta el momento son esperanzadores.

\section{Referencias}

Anastasi, F., Caprilli, S., Grotto, R. P. L., Scollo, M., \& Messeri, A. (2007). Interactive music as a treatment for pain and stress in children during venipuncture: a randomized prospective study. Journal of Developmental \& Behavioral Pediatrics, 28, 399-403. doi:10.1097/ DBP.0b013e31811ff8a7

Balan, R., Bavdekar, S. B., \& Jadhav, S. (2009). Can Indian classical instrumental music reduce pain felt during venepuncture? The Indian Journal of Pediatrics, 76, 469-473. doi: 10.1007/s12098-009-0089-y

Barrera, M. E., Rykov, M. H., \& Doyle, S. L. (2002). The effects of interactive music therapy on hospitalized children with cancer: a pilot study. Psycho-Oncology, 11, 379-388. doi: 10.1002/pon.589 
Bennett, M. P., \& Lengacher, C. A. (2006). Humor and laughter may influence health. I. History and background. Evidence-Based Complementary and Alternative Medicine, 3, 61-63.

Bertini, M., Isola, E., Paolone, G., \& Curcio, G. (2011). Clowns benefit children hospitalized for respiratory pathologies. Evidence-Based Complementary and Alternative Medicine, 8, 1-9. doi: 10.1093/ecam/neq064

Braun, C., Stangler, T., Narveson, J., \& Pettingell, S. (2009). Animal-assisted therapy as a pain relief intervention for children. Complementary Therapies in Clinical Practice, 15, 105109. doi: 10.1016/j.ctcp.2009.02.008

Bruehl, S., Carlson, C. R., \& McCubbin, J. A. (1993). Two brief interventions for acute pain. Pain, 54, 29-36. http://dx.doi.org/10.1016/03043959(93)90096-8

Burgdorf, J., \& Panksepp, J. (2006). The neurobiology of positive emotions. Neuroscience and Biobehavioral Reviews, 30, 173-187.

Calmet, L., Regalado, M., \& Guevara, M. (2008). Influencia de la risoterapia en las características psicológicas y sociales del niño escolar hospitalizado. Revista Enfermería Herediana, 1, 19-25. Recuperado de http:// bases.bireme.br/cgi-bin/wxislind.exe/iah/ online/?IsisScript $=$ iah/iah.xis\&src=google\& base $=$ LILACS\&lang $=p \&$ nextAction $=$ Ink\&ex prSearch $=559627$ \&indexSearch $=I D$

Caprilli, S., \& Messeri, A. (2006). Animal-assisted activity at A. Meyer Children's Hospital: a pilot study. Evidence-based Complementary and Alternative Medicine, 3, 379-383. doi:10.1093/ecam/nel029

Cogan, R., Cogan, D., Waltz, W., \& McCue, M. (1987). Effects of laughter and relaxation on discomfort thresholds. Journal of Behavioral Medicine, 10, 139-144.

Cruz, C. (2009). Mascotas: ¿amigos medicinales?. Alternativas en Psicología, 14, 48-57. Recuperado de http://pepsic. bvsalud.org/scielo.php?pid = S1405339X2009000100005\&script = sci_ arttext\&tlng=es

Danner, D. D., Snowdon, D. A., \& Friesen, W. V. (2001). Positive emotions in early life and longevity: findings from the nun study.
Journal of Personality and Social Psychology, 80, 804-813.

Dowling, J. S., Hockenberry, M., \& Gregory, R. L. (2003). Sense of humor, childhood cancer stressors, and outcomes of psychoosical adjustment, immune function, and infection. Journal of Pediatric Oncology Nursing, 20, 271-292.

Finan, P. H., \& Garland, E. L. (2015). The role of positive affect in pain and its treatment. Clinical Journal of Pain, 31, 177-187.

Frankenfield, P. K. (1996). The power of humor and play as nursing intrventions for a child with cáncer: A case report. Journal of Pediatric Oncology Nursing, 13, 15-20.

Fredrickson, B. L. (2000). Cultivating positive emotions to optimize health and wellbeing. Prevention and Treatment, 3(1). Recuperado de http://psycnet.apa.org/ doi/10.1037/1522-3736.3.1.31a

Fredrickson, B. L. (2003). The value of positive emotions. American Scientist, 91, 330-335.

Golan, G., Tighe, P., Dobija, N., Perel, A., \& Keidan, I. (2008). Clowns for the prevention of preoperative anxiety in children: A randomized controlled trial. Pediatric Anesthesia, 19, 262-266. doi: 10.1111/j.14609592.2008.02903.x

Gottlieb, S. E., \& Portnoy, S. (1988). The role of playin a pediatric bone marrow transplantation unit. Children's Health Care, 16, 177181.

Gutiérrez, M. A., Ortigosa, J. M., Girón, O., Ruiz, R., Sánchez, J., Guirao, M. J.,...Cárceles Barón, M. D. (2008). Evaluación del efecto de la actuación de los payasos de hospital sobre la ansiedad, en los niños sometidos a una intervención quirúrgica. Cirugía Pediátrica, 21, 195-198. Recuperado de http://www.secipe.org/SB10revist.asp?cid=42

Hansen, L. K., Kibaek, M., Martinussen, T., Kragh, L., \& Hejl, M. (2011). Effect of a clown's presence at botulinum toxin injections in children: a randomized, prospective study. Journal of Pain Research, 4, 297. doi: 10.2147/JPR.S23199

Jofré, M. (2005). Visita terapéutica de mascotas en hospitales. Revista Chilena de Infectología, 22, 257-263. doi: 0.4067/S0716- 
10182005000300007

Kaminski, M., Pellino, T., \& Wish, J. (2002). Play and pets: The physical and emotional impact of child-life and pet therapy on hospitalized children. Children's Health Care, 31, 321-335. doi: 10.1207/S15326888CHC3104_5

Kemper, K. J., Hamilton, C. A., McLean, T. W., \& Lovato, J. (2008). Impact of music on pediatric oncology outpatients. Pediatric Research, 64, 105-109. doi: 10.1203/ PDR.0b013e318174e6fb

Kingsnorth, S., Blain, S., \& McKeever, P. (2011). Physiological and emotional responses of disabled children to therapeutic clowns: a pilot study. Evidence-Based Complementary and Alternative Medicine, 8, 1-10. doi: 10.1093/ecam/neq008

Lambert, R. B., \& Lambert, N. K. (1995). The effects of humor on secretory inmnoglobulin. A levels in school-aged children. Pediatric Nursing, 21, 16-19.

Linge, L. (2013). Joyful and serious intentions in the work of hospital clowns: A metaanalysis based on a 7-year research project conducted in three parts. International journal of Qualitative Studies on Health and Wellbeing, 8, 1-8. doi: 10.3402/qhw.v8i0.18907

López-Cepero, J., Rodríguez, L., Perea, M. A., Blanco, N., Tejada, A., \& Blanco, A. (2014). Animal-assisted Interventions: Review of current status and future challenges. International Journal of Psychology and Psychological Therapy, 14, 85-101. Recuperado de http://www.ijpsy.com/volumen14/ num 1/376.html

Lyubomirsky, S., King, L., \& Diener, E. (2005). The benefits of frequent positive affect: Does happiness lead to success? Psychological Bulletin, 131, 803-855.

Maanen, L. V. (2012). The effect of hospital clown visits on hospitalized children: $A$ study into the effects and its measures (Trabajo Fin de Máster de la Universidad de Utrecht). Recuperado de http://dspace.library.uu.nl/ handle/1874/256750

Martin, R. A., \& Lefcourt, H. M. (1983). Sense of humor as a moderator of the relation between stressors and moods. Journal of Personality and Social Psychology, 45, 1313-1324.
Meisel, V., Chellew, K., Ponsell, E., Ferreira, A., Bordas, L., \& García-Banda, G. (2009). El efecto de los «payasos de hospital» en el malestar psicológico y las conductas desadaptativas de niños y niñas sometidos a cirugía menor. Psicothema, 21, 604-609. Recuperado de http://www.psicothema.com/ pdf/3678.pdf

Ortigosa, J. M., Gutierrez, M. A., Astilleros, M. J., Sanchez I., \& Riquelme A. (2011). Los payasos en el hospital: valoración de un programa para reducir la ansiedad ante la cirugía. Revista Iberoamericana de Psicosomática, 99, 41-49. Recuperado de http://dialnet.unirioja.es/servlet/articulo?codigo $=3781329$

Ortiz, A. L.(2006). Ansiedad y miedos en niños ante la hospitalización. Investigación, intervención, programas y técnicas. Revista de la Facultad de Psicología Universidad Cooperativa de Colombia, 3, 84-100. Recuperado de https://www.yumpu.com/es/document/ view/14880631/ansiedad-y-miedos-en-ninos-ante-la-hospitalizacion-universidad-

Pelechano, V. (2008). Enfermedades crónicas y psicología. Madrid: Klinik.

Pinquart, M., Skolaude, D., Zaplinski, K., \& Maier, R. F. (2011). Do clown visits improve psychological and sense of physical well-being of hospitalized pediatric patients? a randomized-controlled trial. Klinische Pädiatrie, 223, 74-78. doi: 10.1055/s-0030-1267932

Ripoll, R., \& Quintana, I. (2010). Risas y terapias positivas: moderno enfoque y aplicaciones prácticas en medicina. Revista de Psiquiatría y Salud Mental, 3, 27-34. doi: http://dx.doi. org/10.1016/S1888-9891(10)70006-9

Rivara, G., Bucher, A., Briceño, D., Huaipar,S., Liu, C., De Souza, K.,...Huando, R. (2010). Clowns como método analgésico no farmacológico en niños hospitalizados. Revista Peruana de Pediatría, 63, 9-19. Recuperado de http://www.pediatriaperu.org/images/ stories/Curso_Internacional/2011/Revista\%20Pediatria\%202010\%20Vol\%2063\%20 $\mathrm{N}-2$.pdf

Rivara, G., Huáipar, S., Liu, C. Briceño, D., Bucher, A., Samamé, J. C.,... Valencia Palpa, Z. (2008). Comparación de tres géneros musicales como método analgésico no farmacológico: 
estudio randomizado ciego simple. Revista Peruana de Pediatría, 61(4), 221-228. Recuperado de http://revistas.concytec.gob.pe/ scielo.php?script=sci_arttext\&pid=S1993$68262008000400005 \& \operatorname{lng}=e n \& n r m=i s o$

Serradas, M. (2006). La música como medio de expresión del niño hospitalizado. Educere, 10, 35-41. Recuperado de http://www. redalyc.org/articulo.oa?id $=35603206$

Sobo, E. J., Eng, B., \& Kassity-Krich, N. (2006). Canine visitation (pet) therapy pilot data on decreases in child pain perception. Journal of Holistic Nursing, 24, 51-57. doi: 10.1177/0898010105280112

Standley, J. M., Cassidy, J., Grant, R., Cevasco, A., Szuch, C., Nguyen, J.,... Adams, K. (2010). The effect of music reinforcement for non-nutritive sucking on nipple feeding of premature infants. Pediatric nursing, 36, 138-145. Recuperado de http:// web.a.ebscohost.com.accedys2.bbtk.ull. es/ehost/detail?sid=34ea0735-3f54-447bab7a-660691c784d9\%40sessionmgr400 $2 \&$ vid $=1 \&$ hid $=4214 \&$ bdata $=J \mathrm{mxhbmc9}$ ZXMmc2I0ZT1laG9zdC1 saXZl\#db=ccm\& $\mathrm{AN}=2010703924$

Stone, A. A., Cox, D. S., Valdimarsdottir, H., Jandorf, L., \& Neale, J. M. (1987). Evidence that secretory IgA antibody is associated with daily mood. Journal of Personality and Social Psychology, 52(5), 988-983.

Tan, S. A., Tan, L. G., Berk, L. S., Lukman, S. T., \& Lukman, L. F. (1997). Mirthful laughter an effective adjunct in cardiac rehabilitation. The Canadian Journal of Cardiology, 13, 190.

Tsai, C. C., Friedmann, E., \& Thomas, S. A. (2010). The effect of animal-assisted therapy on stress responses in hospitalized children. Anthrozoos: A Multidisciplinary Journal of the Interactions of People \& Animals, 23, 245-258. doi: $10.2752 / 175303710 \times 127504$ 51258977

Vagnoli, L., Caprilli, S., \& Messeri, A. (2010). Parental presence, clowns or sedative premedication to treat preoperative anxiety in children: what could be the most promising option? Pediatric Anesthesia, 20, 937-943. doi: 10.1111/j.1460-9592.2010.03403.x

Vagnoli, L., Caprilli, S., Robiglio, A., \& Messeri,
A. (2005). Clown doctors as a treatment for preoperative anxiety in children: a randomized, prospective study. Pediatrics, 116, e563-e567. doi:10.1542/peds.2005-0466

Weisenberg, M., Raz, T., \& Hener, T. (1998). The influence of film-induced mood on pain perception. Pain, 76, 365-375.

Wolyniez, I., Rimon, A., Scolnik, D., Gruber, A., Tavor, O., Haviv, E. et al. (2013). The Effect of a Medical Clown on Pain During Intravenous Access in the Pediatric Emergency Department A Randomized Prospective Pilot Study. Clinical pediatrics, 52, 1168-1172. doi:10.1177/0009922813502257

Wright, J., Adams, D., \& Vohra, S., (2013). Complementary, Holistic, and Integrative Medicine: Music for Procedural Pain. Pediatrics in Review, 34, e42-e46. doi: 10.1542/pir.34$11-\mathrm{e} 42$

Selye, H. (1974). Stress in Health and Disease. Boston: Butterworth.

Wu, A. S., Niedra, R., Pendergast, L., \& McCrindle, B. W. (2002). Acceptability and impact of pet visitation on a pediatric cardiology inpatient unit. Journal of Pediatric Nursing, 17, 354-362. doi: 10.1053/jpdn.2002.127173. 